\title{
MRgFUS of small breast cancer: what should be learned from a case of local recurrence
}

\author{
Hidemi Furusawa*, Junichi Shidooka, Masuko Inomata, Emiko Hirabara, Hiroshi Nakahara, Yukiko Ymaguchi \\ From Current and Future Applications of Focused Ultrasound 2014. 4th International Symposium \\ Washington, D.C, USA. 12-16 October 2014
}

\section{Background/introduction}

Background: The aim of local treatment of breast cancer is to completely eradicate the cancer cells. The excisionless study which is an alternative local treatment for the breast cancer with MRgFUS is going on at our facility from June 2005. Purpose: To validate the safety and efficacy of this local treatment. And to probe the cause of local recurrence.

\section{Methods}

The main inclusion criteria; 1 ) breast cancer diagnosed by the needle biopsy 2) Receptor status confirmation 3) tumor size $15 \mathrm{~mm} \mathrm{4)}$ ) well demarcated mass in contrast enhanced MRI. The main exclusion criteria; 1 ) pure type mucinous carcinoma 2 ) invasive micropapillary carcinoma 3) location of the tumor requires a high sonication angle. The postoperative needle biopsy was performed again within three weeks after ablation and no residual viable cancer cells were identified pathologically. The following radiotherapy should be performed. The patients are followed by three modalities every 3 to 6 months.

\section{Results and conclusions}

Eighty-seven patients were enrolled and 72 breast lesions were treated sequentially. The median age was 56 years old $(29-79)$. The average tumor size was $11.0 \mathrm{~mm}(5-15)$. The average treatment duration was 126 minutes (41-246). The median follow-up period was 68 months (2-108). Thirty-eight cases have been followed up for more than 60 months. Although there were no severe adverse events and any distant recurrence cases, the local recurrence developed seven years after the initial treatment in only one invasive breast cancer case. There are two presumptive causes of this local recurrence. The one reason could be the cancer

Breastopia Clinic, Miyazaki, Japan (http://creativecommons.org/publicdomain/zero/1.0/) applies to the data made available in this article, unless otherwise stated. 